PROCEEDINGS OF THE

AMERICAN MATHEMATICAL SOCIETY

Volume 140, Number 11, November 2012, Pages 3959-3972

S 0002-9939(2012)11248-1

Article electronically published on March 23, 2012

\title{
ON LIMIT BEHAVIOR OF SEMIGROUP ACTIONS ON NONCOMPACT SPACES
}

\author{
JOSINEY A. SOUZA
}

(Communicated by Yingfei Yi)

\begin{abstract}
This paper is produced in response to the questioning of Morse decomposition for semigroup actions on noncompact spaces. We show how the limit behavior can be studied in arbitrary topological spaces by using powerful tools such as the Stone-Cech compactification and shadowing semigroups. We extend Conley's characterization of chain recurrence in terms of attractors from the setting of flows on compact metric spaces to the setting of semigroup actions on any topological space.
\end{abstract}

\section{INTRODUCTION}

The concept of Morse decomposition for semigroup actions on topological spaces has recently been reproduced in [3, 4. Consistent results could be presented on compact spaces, relating Morse decomposition to the concepts of attractor and chain recurrence. Those theorems are extensions of certain results obtained by C. Conley for dynamical systems on compact spaces. The main one characterizes the chain recurrent set in terms of the attractors. The purpose of this paper is to reproduce this theorem to semigroup action on arbitrary topological spaces.

Several papers dealing with flows and semiflows on noncompact spaces have been produced. Hurley [8, 9, 10] has extended Conley's characterization of chain recurrence in terms of attractors from the setting of flows on compact metric spaces to the setting of flows and semiflows on any metric space. Theorems concerning the relationship among attractors, chain recurrence, and Lyapunov functions for discrete flows on arbitrary metric spaces have been presented in [11. Choi-Chu-Park in [6] have improved certain proofs of [10. More recently, Patrão-San-Martin [13] developed the concept of chain recurrence for semiflows on topological spaces and described how their results on Lyapunov functions can be applied to noncompact spaces via Stone-Čech compactification (we also refer to [12]). The present paper follows this line of investigation to transfer results of semigroup actions from compact spaces to noncompact spaces.

The concept of a shadowing semigroup is another powerful tool for studying chain recurrence for semigroup actions. Braga Barros and San Martin [1 have introduced shadowing semigroups to study chain control sets for semigroup actions on compact metric spaces. This tool has earlier been used to describe the chain transitivity of flows and semiflows on fiber bundles and topological spaces (see 2, 13, 14]). We also apply this methodology in this paper. Semigroup actions on

Received by the editors February 10, 2011 and, in revised form, May 12, 2011.

2010 Mathematics Subject Classification. Primary 37B35, 37B25.

Key words and phrases. Semigroup actions, chain recurrence, Morse decompositions. 
noncompact homogeneous spaces is the main setting where we obtain consistent results.

In Section 2 we give the main definitions of semigroup actions and introduce the notion of Morse decomposition on topological spaces not necessarily compact. For studying Morse decomposition on noncompact spaces it is convenient to consider its dynamical version since it does not depend on attractor-repeller pairs. Dynamic Morse decomposition for semigroup action on compact spaces has been introduced in [5, and it can be adapted to noncompact spaces (see Definition 4).

In Section 3 we study the limit behavior of semigroup action on topological spaces via their compactifications. We illustrate how the results of [3] can be applied to noncompact spaces in this way. Since limit sets in noncompact spaces may be empty, we explore the actions extended to the Stone-Čech compactifications. In this way we can link chain recurrence and attractors even on noncompactness (compare Proposition 2 and Theorem 2).

In Section 4 we go into the investigation of the special case of semigroup actions on noncompact homogeneous spaces. We can describe the chain recurrence from another point of view by using the concept of a shadowing semigroup. This way of describing chains is powerful whenever local transitivity exists. For a homogeneous space $G / H$, where $G$ is a topological group and $H \subset G$ is a closed subgroup, we define naturally an admissible family of open coverings of $G / H$ from a basis of symmetric neighborhoods at the identity $e$ of $G$, and show that the group $G$ is locally transitive on $G / H$. Then, maximal chain transitive sets are described as intersections of control sets of shadowing semigroups (see Theorem 5). It implies a precise description for maximal chain transitive sets in regular adjoint orbits of Lie groups, which are homogeneous spaces. We use the results of Verdi-Rocio-San Martin [15] to show that the maximal chain transitive sets can be parameterized by the Weyl group.

\section{Semigroup aCtions}

This section contains basic definitions and results of semigroup actions that are treated in this paper. We emphasize the definition of Morse decomposition adapted to topological spaces not necessarily compact.

Let $X$ be a noncompact topological space and let $S$ be a semigroup. An action of $S$ on $X$ is a mapping

$$
\begin{array}{cccc}
\mu: S \times X & \rightarrow & X \\
(s, x) & \mapsto & \mu(s, x)=s x
\end{array}
$$

satisfying $s(t x)=(s t) x$ for all $x \in X$ and $s, t \in S$. We often indicate the semigroup action as $(S, X, \mu)$, or simply $(S, X)$. We denote by $\mu_{s}: X \rightarrow X$ the map $\mu_{s}(\cdot)=$ $\mu(s, \cdot)$. In this paper we assume that $\mu_{s}$ is continuous for all $s \in S$.

For subsets $Y \subset X$ and $A \subset S$ we define

$$
A Y=\bigcup_{s \in A} \mu_{s}(Y) \quad \text { and } \quad A^{*} Y=\bigcup_{s \in A} \mu_{s}^{-1}(Y) .
$$

It is usual to say that $Y$ is forward invariant if $S Y \subset Y$, it is backward invariant if $S^{*} Y \subset Y$, and it is invariant if it is forward and backward invariant.

For establishing a direction to asymptotic behavior of a semigroup action $(S, X)$, we choose a family $\mathcal{F}$ of subsets of $S$ that is a filter basis on the subsets of $S$ (that is, 
$\varnothing \notin \mathcal{F}$ and given $A, B \in \mathcal{F}$ there is $C \in \mathcal{F}$ with $C \subset A \cap B)$ and satisfies the following right translation hypothesis:

For all $s \in S$ and $A \in \mathcal{F}$ there is $B \in \mathcal{F}$ with $B \subset A s$.

We refer to papers [3, 4, for usual families satisfying these properties.

Definition 1. The $\omega$-limit set of $Y \subset X$ for the family $\mathcal{F}$ is defined as

$$
\omega(Y, \mathcal{F})=\bigcap_{A \in \mathcal{F}} \operatorname{cls}(A Y) .
$$

The $\omega^{*}$-limit set of $Y \subset X$ is defined as

$$
\omega^{*}(Y, \mathcal{F})=\bigcap_{A \in \mathcal{F}} \operatorname{cls}\left(A^{*} Y\right)
$$

An important class of limit set consists of attractors and repellers, as follows.

Definition 2. An $\mathcal{F}$-attractor for $(S, X)$ is a set $\mathcal{A}$ that admits a neighborhood $V$ such that $\omega(V, \mathcal{F})=\mathcal{A}$. The neighborhood $V$ is called an attractor neighborhood of $\mathcal{A}$. An $\mathcal{F}$-repeller is a set $\mathcal{R}$ that has a neighborhood $U$ with $\omega^{*}(U, \mathcal{F})=\mathcal{R}$. The neighborhood $U$ is called a repeller neighborhood of $\mathcal{R}$. We consider both the empty set and $X$ as attractors and repellers.

The following important result has been proved in [3, Proposition 3.1].

Proposition 1. Assume that $X$ is a compact Hausdorff space. For each $\mathcal{F}$-attractor $\mathcal{A}$ with attractor neighborhood $V$ there is some $A \in \mathcal{F}$ such that $\operatorname{cls}(A V) \subset \operatorname{int}(V)$. For each $\mathcal{F}$-repeller $\mathcal{R}$ with repeller neighborhood $U$ there is some $A \in \mathcal{F}$ such that $\operatorname{cls}\left(A^{*} U\right) \subset \operatorname{int}(U)$.

Let $\mathcal{A}$ be an $\mathcal{F}$-attractor in $X$. We define the set

$$
\mathcal{A}^{*}=\{x \in X: \omega(x, \mathcal{F}) \cap \mathcal{A}=\emptyset\} .
$$

We call $\mathcal{A}^{*}$ the complementary repeller of $\mathcal{A}$, and $\left(\mathcal{A}, \mathcal{A}^{*}\right)$ an attractor-repeller pair. Notice that $\mathcal{A}$ and $\mathcal{A}^{*}$ are disjoint. From [3, Proposition 3.4], $\mathcal{A}^{*}$ is invariant and coincides with the set $X \backslash\{x \in X: \omega(x, \mathcal{F}) \subset \mathcal{A}\}$.

Definition 3. A subset $Y$ of $X$ is called isolated invariant for the action of the semigroup $S$ if it is invariant and there is a neighborhood $V$ of $Y$ such that, for $x \in V, S x \subset V$ and $S^{*} x \subset V$ imply $x \in Y$.

Definition 4. A dynamic $\mathcal{F}$-Morse decomposition for $(S, X)$ is a finite collection $\mathcal{M}=\left\{\mathcal{C}_{i}, i=1, \ldots, n\right\}$ of nonempty, pairwise disjoint, isolated invariant and closed sets such that:

(1) For all $x \in X$ one has $\omega(x, \mathcal{F}), \omega^{*}(x, \mathcal{F}) \subset \bigcup_{i=1}^{n} \mathcal{C}_{i}$.

(2) (No-cycle condition) Suppose there are ${ }^{i=1} \mathcal{C}_{j_{0}}, \mathcal{C}_{j_{1}}, \ldots, \mathcal{C}_{j_{l}}$ and $x_{1}, \ldots, x_{l} \in$ $X \backslash \bigcup_{i=1}^{n} \mathcal{C}_{i}$ with $\varnothing \neq \omega^{*}\left(x_{k}, \mathcal{F}\right) \subset \mathcal{C}_{j_{k-1}}$ and $\varnothing \neq \omega\left(x_{k}, \mathcal{F}\right) \subset \mathcal{C}_{j_{k}}$, for $k=$ $1, \ldots, l$; then $\mathcal{C}_{j_{0}} \neq \mathcal{C}_{j_{l}}$.

We notice that the definition of dynamic Morse decomposition presented here coincides with the one for semigroup actions on compact spaces introduced in $[5]$. Moreover, dynamic $\mathcal{F}$-Morse decomposition can be obtained by means of attractors. In fact, for an increasing sequence of $\mathcal{F}$-attractors $\emptyset=\mathcal{A}_{0} \subset \mathcal{A}_{1} \subset \cdots \subset \mathcal{A}_{n}=X$, 
let $\mathcal{C}_{i}=\mathcal{A}_{i} \cap \mathcal{A}_{i-1}^{*}, i=1, \ldots, n$. The ordered collection $\mathcal{M}=\left\{\mathcal{C}_{1}, \ldots, \mathcal{C}_{n}\right\}$ is a dynamic $\mathcal{F}$-Morse decomposition for $(S, X)$, and each set $\mathcal{C}_{i}$ is called an $\mathcal{F}$-Morse set.

Let us see some examples of Morse decomposition for general semigroup action on noncompact spaces.

Example 1. Let $\mathcal{V}$ be a family of vector fields on a noncompact $n$-dimensional manifold $X$. Consider the control system on $X$ determined by the set of vector fields $\mathcal{V}$, that is, the control system such that its trajectories are concatenations of trajectories of vectors in $\mathcal{V}$. These trajectories are determined by the system semigroup

$$
S=\left\{\mathrm{e}^{t_{n} Y_{n}} \mathrm{e}^{t_{n-1} Y_{n-1}} \ldots \mathrm{e}^{t_{0} Y_{0}}: Y_{j} \in \mathcal{V}, t_{j} \geq 0, n \in \mathbb{N}\right\},
$$

which is a semigroup of diffeomorphisms of $X$. Then, the control system is the semigroup action $(S, X)$. For each $t>0$ we define the set

$$
U(t)=\left\{\mathrm{e}^{t_{n} Y_{n}} \mathrm{e}^{t_{n-1} Y_{n-1}} \ldots \mathrm{e}^{t_{0} Y_{0}}: Y_{j} \in \mathcal{V}, \sum_{j=0}^{n} t_{j} \geq t, n \in \mathbb{N}\right\}
$$

and fix the family $\mathcal{F}=\{U(t): t>0\}$. The limit behavior with respect to $\mathcal{F}$ establishes the limit behavior of the system (see [3, 5]). The limit sets of a subset $V \subset X$ are

$$
\omega(V, \mathcal{F})=\bigcap_{t>0} \operatorname{cls}(U(t) V) \quad \text { and } \quad \omega^{*}(V, \mathcal{F})=\bigcap_{t>0} \operatorname{cls}\left(U(t)^{-1} V\right) .
$$

Consider, for instance, the special case $\mathcal{V}=\{F, G\}$, where $F=\left(F_{1}, F_{2}\right), G=$ $\left(G_{1}, G_{2}\right)$ are two vector fields on $X=\mathbb{R}^{2}$ given by

$$
\begin{gathered}
F_{1}(x)=-x_{2}+x_{1}\|x\|^{2} \sin \frac{\pi}{\|x\|}, \quad F_{2}(x)=x_{1}+x_{2}\|x\|^{2} \sin \frac{\pi}{\|x\|}, \\
G_{1}(x)=\alpha(x) x_{1}+(\alpha(x)-\beta(x)) x_{2}+x_{1}\|x\|^{2}\left(\beta(x) \sin \frac{\pi}{\|x\|}-4 \alpha(x)\right), \\
G_{2}(x)=\alpha(x) x_{2}+(\beta(x)-\alpha(x)) x_{1}+x_{2}\|x\|^{2}\left(\beta(x) \sin \frac{\pi}{\|x\|}-4 \alpha(x)\right),
\end{gathered}
$$

and $F(0)=G(0)=0$, where

$$
\alpha(x)=\left\{\begin{array}{ll}
1 & \text { if }\|x\| \leq 1 / 2 \\
0 & \text { if }\|x\|>1 / 2
\end{array}, \quad \beta(x)=\left\{\begin{array}{cc}
0 & \text { if }\|x\| \leq 1 / 2 \\
1 & \text { if }\|x\|>1 / 2
\end{array} .\right.\right.
$$

In other words, for $\|x\| \leq 1 / 2$, the vector field $G$ is $G_{1}(x)=x_{2}+x_{1}\left(1-4\|x\|^{2}\right)$ and $G_{2}(x)=-x_{1}+x_{2}\left(1-4\|x\|^{2}\right)$, and for $\|x\|>1 / 2, G$ coincides with $F$. The sets $\mathcal{C}_{1}=\{x:\|x\| \geq 1\}$ and $\mathcal{C}_{2}=\{x:\|x\| \leq 1 / 2\}$ are isolated invariant and closed. Furthermore, for $x \in M \backslash\left(\mathcal{C}_{1} \cup \mathcal{C}_{2}\right)$ we have $\omega^{*}(x, \mathcal{F}) \subset \mathcal{C}_{1}$ and $\omega(x, \mathcal{F}) \subset \mathcal{C}_{2}$. Thus, $\mathcal{M}=\left\{\mathcal{C}_{1}, \mathcal{C}_{2}\right\}$ is a dynamic Morse decomposition of the system (see Figure 1).

Example 2. Suppose that $S \subset \mathbb{R}^{n}$ is a cone and $\mu: S \times X \rightarrow X$ is an action of $S$ on a topological space $X$. Consider an ordered basis $u_{1}, \ldots, u_{n}$ for $\mathbb{R}^{n}$. We denote this action by $\mu\left(\left(t_{1}, \ldots, t_{n}\right), x\right)=\left(t_{1}, \ldots, t_{n}\right) \cdot x$. For an $i \in\{1, \ldots, n\}$ and $t>0$ we consider the subset

$$
A_{i}(t)=\left\{\left(t_{1}, \ldots, t_{n}\right) \in S: t_{i} \geq t\right\}
$$




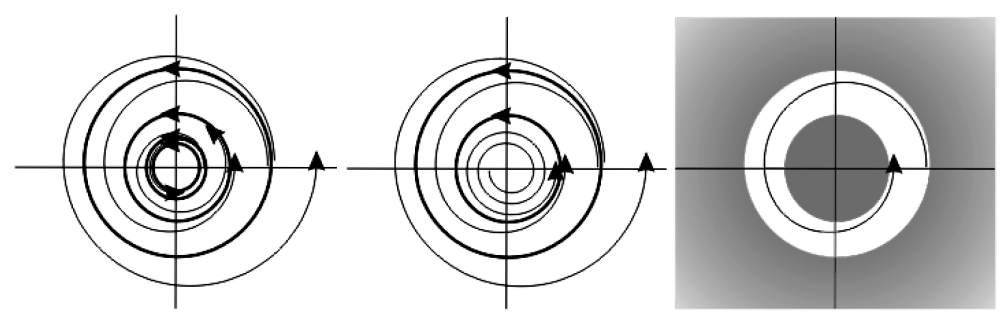

Figure 1. Trajectories of $F$ and $G$, and the Morse decomposition.

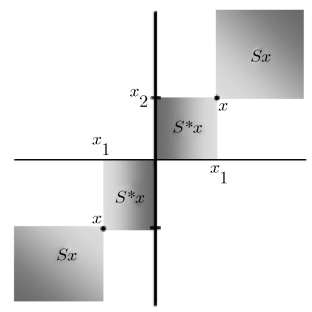

FiguRE 2. Orbits, limit sets, and the Morse decomposition of the cone $S$.

and define the family $\mathcal{F}_{i}=\left\{A_{i}(t): t>0\right\}$. The $\omega$-limit set of a subset $V \subset X$ is

$$
\omega\left(V, \mathcal{F}_{i}\right)=\left\{\begin{array}{c}
x \in X: \text { there is a net }\left(t_{1}^{k}, \ldots, t_{n}^{k}\right) \cdot x_{k} \text { with } t_{i}^{k} \rightarrow+\infty \\
\text { such that }\left(t_{1}^{k}, \ldots, t_{n}^{k}\right) \cdot x_{k} \rightarrow x
\end{array}\right\} .
$$

For instance, assume that $S=\left\{\left(x_{1}, x_{2}\right) \in \mathbb{R}^{2}: x_{1}, x_{2} \geq 0\right\}, X=\mathbb{R}^{2}$, and $\left(t_{1}, t_{2}\right)$. $\left(x_{1}, x_{2}\right)=\left(e^{t_{1}} x_{1}, e^{t_{2}} x_{2}\right)$. Fix the family $\mathcal{F}_{1}$ for limit behavior. The origin $(0,0)$ is a fixed point of $S$. For $x=\left(x_{1}, x_{2}\right) \in X$, with $x_{1}, x_{2} \neq 0$, we have $\omega\left(x, \mathcal{F}_{1}\right)=\varnothing$, $\omega^{*}\left(x, \mathcal{F}_{1}\right)=\{0\} \times\left[0, x_{2}\right]$ if $x_{2}>0$, and $\omega^{*}\left(x, \mathcal{F}_{1}\right)=\{0\} \times\left[x_{2}, 0\right]$ if $x_{2}<0$. For $x=\left(0, x_{2}\right) \in X$, with $x_{2} \neq 0$, we have $\omega\left(x, \mathcal{F}_{1}\right)=\{0\} \times\left[x_{2},+\infty\right)$ and $\omega^{*}\left(x, \mathcal{F}_{1}\right)=$ $\{0\} \times\left[0, x_{2}\right]$ if $x_{2}>0 ; \omega\left(x, \mathcal{F}_{1}\right)=\{0\} \times\left(-\infty, x_{2}\right]$ and $\omega^{*}\left(x, \mathcal{F}_{1}\right)=\{0\} \times\left[x_{2}, 0\right]$ if $x_{2}<0$. Finally, for $x=\left(x_{1}, 0\right) \in X$, with $x_{1} \neq 0$, we have $\omega\left(x, \mathcal{F}_{1}\right)=\varnothing$ and $\omega^{*}\left(x, \mathcal{F}_{1}\right)=\{(0,0)\}$. Thus, $\mathcal{M}=\{\{0\} \times(-\infty,+\infty)\}$ is a dynamic Morse decomposition of $(S, X)$ (see Figure 2).

Note that the Morse decomposition in Example 2 consists of only one element. This fact has occurred since there were empty limit sets. In general, it happens on the setting of semigroup actions on noncompact spaces or on the setting of $(S, X, \mu)$ where the maps $\mu_{s}: X \rightarrow X$ are not surjective. However, a Morse decomposition is useful to describe the limit behavior in any of those situations.

2.1. Chain recurrence. The concept of chain recurrence for the semigroup action $(S, X)$ depends on an admissible family of open coverings of $X$. Let $\mathcal{U}$ and $\mathcal{V}$ be open coverings of $X$. We write $\mathcal{V} \leqslant \mathcal{U}$ if $\mathcal{V}$ is a refinement of $\mathcal{U}$. We write $\mathcal{V} \leqslant \frac{1}{2} \mathcal{U}$ if for every $V, V^{\prime} \in \mathcal{V}$, with $V \cap V^{\prime} \neq \emptyset$, there is $U \in \mathcal{U}$ such that $V \cup V^{\prime} \subset U$. For an open covering $\mathcal{U}$ of $X$ and a compact subset $K \subset X$, we denote

$$
[\mathcal{U}, K]=\{U \in \mathcal{U}: K \cap U \neq \emptyset\} .
$$


Let $Y \subset X$ be an open subset and suppose that $K \subset Y$ is a compact subset of $X$. An open covering $\mathcal{U}$ of $X$ is called $K$-subordinated to $Y$ if for any $U \in[\mathcal{U}, K]$ one has $U \subset Y$.

Definition 5. A family $\mathcal{O}$ of open coverings of $X$ is said to be admissible if it satisfies the following properties:

(1) For each $\mathcal{U} \in \mathcal{O}$, there is an open covering $\mathcal{V} \in \mathcal{O}$ such that $\mathcal{V} \leqslant \frac{1}{2} \mathcal{U}$.

(2) Let $Y \subset X$ be an open set and $K$ a compact subset of $X$ contained in $Y$. Then there is an open covering $\mathcal{U} \in \mathcal{O}$ that is $K$-subordinated to $Y$.

(3) For any $\mathcal{U}, \mathcal{V} \in \mathcal{O}$, there is $\mathcal{W} \in \mathcal{O}$ such that $\mathcal{W} \leqslant \mathcal{U}$ and $\mathcal{W} \leqslant \mathcal{V}$.

It is well known that the family of all open coverings is admissible if $X$ is paracompact (see [16, Section 20]).

Definition 6. For $x, y \in X$, an open cover $\mathcal{U}$ of $X$ and $A \subset S$, we define a $(\mathcal{U}, A)$ chain from $x$ to $y$ as a sequence $x_{0}=x, x_{1}, \ldots, x_{n}=y$ in $X, a_{0}, \ldots, a_{n-1} \in A$ and open sets $U_{0}, \ldots, U_{n-1} \in \mathcal{U}$ such that $a_{i} x_{i}, x_{i+1} \in U_{i}$, for all $i=0, \ldots, n-1$.

Definition 7. Let $\mathcal{O}$ be a family of open coverings of $X$ and $\mathcal{F}$ a family of subsets of $S$. Given a nonempty subset $Y \subset X, A \in \mathcal{F}$, and $\mathcal{U} \in \mathcal{O}$ we define the $\Omega$-chain limit set of $Y$ as

$$
\Omega_{\mathcal{O}, \mathcal{F}}(Y)=\bigcap_{\mathcal{U} \in \mathcal{O}, A \in \mathcal{F}} \Omega(Y, \mathcal{U}, A),
$$

where $\Omega(Y, \mathcal{U}, A)=\{y \in X$ : there are $x \in Y$ and a $(\mathcal{U}, A)$-chain from $x$ to $y\}$, and define the $\Omega^{*}$-chain limit set of $Y$ as

$$
\Omega_{\mathcal{O}, \mathcal{F}}^{*}(Y)=\bigcap_{\mathcal{U} \in \mathcal{O}, A \in \mathcal{F}} \Omega^{*}(Y, \mathcal{U}, A)
$$

where $\Omega^{*}(Y, \mathcal{U}, A)=\{y \in X$ : there are $x \in Y$ and a $(\mathcal{U}, A)$-chain from $y$ to $x\}$.

A subset $Y \subset X$ is $\mathcal{F}$-chain transitive if for all $y \in Y, Y \subset \Omega_{\mathcal{O}, \mathcal{F}}(y)$. A point $x \in X$ is $\mathcal{F}$-chain recurrent if $x \in \Omega_{\mathcal{O}, \mathcal{F}}(x)$. We denote by $\mathfrak{R}$ the $\mathcal{F}$-chain recurrence set, that is, the set of all $\mathcal{F}$-chain recurrent points.

Remark 1. (1) It is not difficult to see that $\Omega(Y, \mathcal{U}, A)$ and $\Omega^{*}(Y, \mathcal{U}, A)$ are open sets of $X$.

(2) The maximal (with respect to set inclusion) $\mathcal{F}$-chain transitive sets are given by

with $x \in \mathfrak{R}$.

$$
\mathcal{M}_{x}=\Omega_{\mathcal{O}, \mathcal{F}}(x) \cap \Omega_{\mathcal{O}, \mathcal{F}}^{*}(x)
$$

(3) The $\mathcal{F}$-chain recurrence set $\mathfrak{R}$ and the maximal $\mathcal{F}$-chain transitive sets are closed (see [3, Propositions 4.6 and 4.7]).

The next result has been proved in 3, Theorem 4.1]. It is the extension of Conley's characterization of chain recurrence in terms of attractors from the setting of flows on compact metric spaces to the setting of semigroup actions on compact spaces.

Proposition 2. Assume that $X$ is a compact Hausdorff space. Then the $\mathcal{F}$-chain recurrence set $\mathfrak{R}$ is the set

$$
\bigcap\left\{\mathcal{A} \cup \mathcal{A}^{*}: \mathcal{A} \text { is an } \mathcal{F} \text {-attractor }\right\},
$$

where $\mathcal{A}^{*}$ is the complementary repeller of $\mathcal{A}$. 


\section{Stone-ČECH COMPACTIFICATion}

In this section we show how the Stone-Čech compactification can be used to study the limit behavior on noncompact spaces. The natural idea is to explore the actions extended to the compactifications and to apply the results from the semigroup action on compact spaces.

Let $(S, X, \mu)$ be a semigroup action, where $X$ is a Tychonoff space. Let $\beta X$ be the Stone-Cech compactification of $X$, with imbedding $e: X \rightarrow \beta X$. For each $s \in S$, there is a unique continuous extension $\beta \mu_{s}: \beta X \rightarrow \beta X$ of $\mu_{s}$ such that $\beta \mu_{s} \circ e=e \circ \mu_{s}$. Then we have the action $\beta \mu: S \times \beta X \rightarrow \beta X$ of $S$ on $\beta X$ such that the following diagram is commutative:

$$
\begin{array}{ccc}
S \times \beta X & \stackrel{\beta \mu}{\rightarrow} & \beta X \\
i d \uparrow e & & \uparrow e \\
S \times X & \mapsto & X
\end{array}
$$

Notice that the imbedding $e$ is a homomorphism of $(S, X, \mu)$ into $(S, \beta X, \beta \mu)$. Thus, if $Y \subset X$ is an invariant set, then $e(Y) \subset \beta X$ is also an invariant set. On the other hand, if $Z \subset \beta X$ is an invariant set, then $e^{-1}(Z \cap e(X))$ is an invariant set in $X$.

It is usual to identify $X$ with its homeomorphic image $e(X) \subset \beta X$.

We choose and fix a family $\mathcal{F}$ of subsets of $S$ which is a filter basis and satisfies the right translation hypothesis. For convenience, we denote by $\omega_{X}(V, \mathcal{F})$ and $\omega_{X}^{*}(V, \mathcal{F})$ the limit sets of $V \subset X$ with respect to $(S, X)$, respectively, that is, $\omega_{X}(V, \mathcal{F})=\bigcap_{A \in \mathcal{F}} \operatorname{cls}_{X}(A V)$ and $\omega_{X}^{*}(V, \mathcal{F})=\bigcap_{A \in \mathcal{F}} \operatorname{cls}_{X}\left(A^{*} V\right)$.

The following result shows that attractors and repellers from $(S, \beta X)$ induce attractors and repellers to $(S, X)$, respectively.

Proposition 3. If $\mathcal{A} \subset \beta X$ is an $\mathcal{F}$-attractor of $(S, \beta X)$, then $\mathcal{A}_{X}=\mathcal{A} \cap X$ is an $\mathcal{F}$-attractor of $(S, X)$. If $\mathcal{R} \subset \beta X$ is an $\mathcal{F}$-repeller of $(S, \beta X)$, then $\mathcal{R}_{X}=\mathcal{R} \cap X$ is an $\mathcal{F}$-repeller of $(S, X)$. Furthermore, $(\mathcal{A} \cap X)^{*}=\mathcal{A}^{*} \cap X$.

Proof. The result is immediate if $\mathcal{A}_{X}$ is empty. Suppose that $\mathcal{A}_{X} \neq \varnothing$. Let $U \subset \beta X$ be an open attractor neighborhood of $\mathcal{A}$. From Proposition 1 there is $B \in \mathcal{F}$ such that $\operatorname{cls}_{\beta X}(B U) \subset U$. Then

$$
\begin{aligned}
\omega_{X}(U \cap X, \mathcal{F}) & =\bigcap_{A \in \mathcal{F}} \operatorname{cls}_{X}(A(U \cap X))=\bigcap_{A \in \mathcal{F}} \operatorname{cls}_{\beta X}(A(U \cap X)) \cap X \\
& \subset \operatorname{cls}_{\beta X}(B U) \cap X \subset U \cap X .
\end{aligned}
$$

Hence, $\omega_{X}(U \cap X, \mathcal{F})$ is an $\mathcal{F}$-attractor in $X$ with attractor neighborhood $U \cap$ $X$. It remains to show that $\mathcal{A}_{X}=\omega_{X}(U \cap X, \mathcal{F})$. Indeed, it is immediate that $\omega_{X}(U \cap X, \mathcal{F}) \subset \mathcal{A}_{X}$. On the other hand, take $x \in \mathcal{A}_{X}$ and let $V \subset X$ be an open neighborhood of $x$ in $X$. There is an open set $N \subset \beta X$ such that $V=N \cap X$. For $A \in \mathcal{F}$, we have $A U \cap N \neq \varnothing$, that is, $U \cap A^{*} N \neq \varnothing$. Since $U \cap A^{*} N$ is an open set and $X$ is dense in $\beta X$, it follows that $U \cap X \cap A^{*} N \neq \varnothing$, that is, $A(U \cap X) \cap V \neq \varnothing$. Hence, $x \in \operatorname{cls}_{X}(A(U \cap X))$, whence $\mathcal{A}_{X} \subset \omega_{X}(U \cap X, \mathcal{F})$ and $\mathcal{A}_{X}=\omega_{X}(U \cap X, \mathcal{F})$. Now, suppose that $\mathcal{R}$ is nonempty. Let $U \subset \beta X$ be an open repeller neighborhood of $\mathcal{R}$. We use Proposition 1 again to show that $\omega_{X}^{*}(U \cap X, \mathcal{F})$ is an $\mathcal{F}$-repeller in $X$. It is immediate that $\omega_{X}^{*}(U \cap X, \mathcal{F}) \subset \mathcal{R}_{X}$. 
On the other hand, take $x \in \mathcal{R}_{X}$ and let $V \subset X$ be an open neighborhood of $x$. There is an open set $N \subset \beta X$ such that $V=N \cap X$. For $A \in \mathcal{F}$, we have $N \cap A^{*} U \neq \varnothing$. Since $X$ is dense in $\beta X$ and $N \cap A^{*} U$ is an open set, it follows that $N \cap X \cap A^{*} U \neq \varnothing$. Hence, $V \cap A^{*}(U \cap X) \neq \varnothing$, and $x \in \operatorname{cls}_{X}\left(A^{*}(U \cap X)\right)$. Thus, $\mathcal{R}_{X} \subset \omega_{X}^{*}(U \cap X, \mathcal{F})$. Finally, if $x \in(\mathcal{A} \cap X)^{*}$, we have $\omega_{X}(x, \mathcal{F}) \nsubseteq \mathcal{A} \cap X$. This means that $\omega_{X}(x, \mathcal{F}) \nsubseteq \mathcal{A}$. Hence, $\omega(x, \mathcal{F}) \nsubseteq \mathcal{A}$, that is, $x \in \mathcal{A}^{*}$. Thus, $(\mathcal{A} \cap X)^{*} \subset \mathcal{A}^{*} \cap X$. On the other hand, if $y \in \mathcal{A}^{*} \cap X$, then $\omega(y, \mathcal{F}) \cap \mathcal{A}=\emptyset$. Hence, $\omega_{X}(y, \mathcal{F}) \cap \mathcal{A} \cap X=\emptyset$, that is, $y \in(\mathcal{A} \cap X)^{*}$. Thus, $\mathcal{A}^{*} \cap X \subset(\mathcal{A} \cap X)^{*}$.

Now, we show that a dynamic $\mathcal{F}$-Morse decomposition in $\beta X$ induces a dynamic $\mathcal{F}$-Morse decomposition in $X$.

Proposition 4. Let $\mathcal{M}=\left\{\mathcal{C}_{1}, \ldots, \mathcal{C}_{n}\right\}$ be a dynamic $\mathcal{F}$-Morse decomposition for $(S, \beta X)$. Define $\mathcal{M}_{X}=\left\{\mathcal{C}_{1} \cap X, \ldots, \mathcal{C}_{n} \cap X\right\}$ where only those $\mathcal{C}_{i} \cap X \neq \varnothing$ are considered, and assume that $\mathcal{M}_{X} \neq\{\varnothing\}$. Then $\mathcal{M}_{X}$ is a dynamic $\mathcal{F}$-Morse decomposition for $(S, X)$.

Proof. It is immediate that $\mathcal{M}_{X}$ is a finite collection of nonempty, pairwise disjoint, isolated invariant and closed sets in $X$. For $x \in X$, we have $\omega_{X}^{*}(x, \mathcal{F}), \omega_{X}(x, \mathcal{F}) \subset$ $\left(\bigcup_{i=1}^{n} \mathcal{C}_{i}\right) \cap X=\bigcup_{i=1}^{n} \mathcal{C}_{i} \cap X$. Suppose there are $\mathcal{C}_{j_{0}}, \mathcal{C}_{j_{1}}, \ldots, \mathcal{C}_{j_{l}}$ and $x_{1}, \ldots, x_{l} \in$ $X \backslash \bigcup_{i=1}^{n} \mathcal{C}_{i} \cap X$ with $\varnothing \neq \omega_{X}^{*}\left(x_{k}, \mathcal{F}\right) \subset \mathcal{C}_{j_{k-1}} \cap X$ and $\varnothing \neq \omega_{X}\left(x_{k}, \mathcal{F}\right) \subset \mathcal{C}_{j_{k}} \cap X$ for $k=1, \ldots, l$. It follows that $\omega^{*}\left(x_{k}, \mathcal{F}\right) \subset \mathcal{C}_{j_{k-1}}$ and $\left.\omega\left(x_{k}\right), \mathcal{F}\right) \subset \mathcal{C}_{j_{k}}$ for $k=1, \ldots, l$. Hence, $\mathcal{C}_{j_{0}} \neq \mathcal{C}_{j_{l}}$, so they are disjoint, and so certainly $\mathcal{C}_{j_{0}} \cap X \neq \mathcal{C}_{j_{l}} \cap X$. Therefore, $\mathcal{M}_{X}$ is a dynamic $\mathcal{F}$-Morse decomposition in $X$.

3.1. Chain recurrence. Let $\mathcal{O}$ be the family of all open coverings of $\beta X$. The family $\mathcal{O}$ induces naturally a family of open coverings in $X$, as follows. For each $\mathcal{U} \in \mathcal{O}$, we define

$$
\mathcal{U}_{X}=\{U \cap X: U \in \mathcal{U}\}
$$

Then we have the family of open coverings in $X$,

$$
\mathcal{O}_{X}=\left\{\mathcal{U}_{X}: \mathcal{U} \in \mathcal{O}\right\}
$$

Since $\mathcal{O}$ is an admissible family of open coverings of $\beta X, \mathcal{O}_{X}$ is an admissible family of open coverings of $X$ (see [13, Proposition 3.19]).

The next result relates the maximal $\mathcal{F}$-chain transitive sets for $(S, X)$ and $(S, \beta X)$.

Theorem 1. A nonempty subset $M \subset X$ is a maximal $\mathcal{F}$-chain transitive set in $X$ if, and only if, there is a maximal $\mathcal{F}$-chain transitive set $N$ in $\beta X$ such that $M=N \cap X$.

Proof. Suppose that $N \subset \beta X$ is a maximal $\mathcal{F}$-chain transitive set and $M=N \cap X \neq$ $\varnothing$. For $x, y \in M, A \in \mathcal{F}$, and $\mathcal{U}_{X} \in \mathcal{O}_{X}$, there are points $u_{0}=x, u_{1}, \ldots, u_{n}=$ $y$ in $\beta X$, elements $a_{0}, \ldots, a_{n-1} \in A$, and open sets $U_{0}, \ldots, U_{n-1} \in \mathcal{U}$ such that $\beta \mu\left(a_{i}, u_{i}\right), u_{i+1} \in U_{i}$, for $i=0, \ldots, n-1$. Then, $u_{i} \in U_{i-1} \cap \beta \mu_{a_{i}}^{-1}\left(U_{i}\right)$ for $i=$ $0, \ldots, n-1$. Since $X$ is dense in $\beta X$, the open sets $U_{i-1} \cap \beta \mu_{a_{i}}^{-1}\left(U_{i}\right)$ intersect $X$. Hence, there are $x_{0}=x, x_{1}, \ldots, x_{n}=y$ in $X, a_{0}, \ldots, a_{n-1} \in A$, and $U_{0} \cap X, \ldots, U_{n-1} \cap$ $X \in \mathcal{U}_{X}$ such that $\mu\left(a_{i}, x_{i}\right), x_{i+1} \in U_{i} \cap X$, for $i=0, \ldots, n-1$. Therefore, $M$ is an $\mathcal{F}$-chain transitive set in $X$. Now, there is a maximal $\mathcal{F}$-chain transitive set $M^{\prime} \subset X$ with $M \subset M^{\prime}$. For $x, y \in M^{\prime}, A \in \mathcal{F}$, and $\mathcal{U} \in \mathcal{O}$, there are $x_{0}=x, x_{1}, \ldots, x_{n}=y$ in $X, a_{0}, \ldots, a_{n-1} \in A$, and $U_{0}, \ldots, U_{n-1} \in \mathcal{U}$ such that $\mu\left(a_{i}, x_{i}\right), x_{i+1} \in U_{i} \cap X$, for $i=0, \ldots, n-1$, that is, $\beta \mu\left(a_{i}, x_{i}\right), x_{i+1} \in U_{i}$, for $i=0, \ldots, n-1$. Hence, $M^{\prime}$ 
is an $\mathcal{F}$-chain transitive set in $\beta X$. It follows that there is a maximal $\mathcal{F}$-chain transitive set $N^{\prime} \subset \beta X$ with $M^{\prime} \subset N^{\prime}$. Since $M \subset N \cap N^{\prime}$, we have $N=N^{\prime}$, and $M^{\prime} \subset X \cap N=M$. Therefore, $M=M^{\prime}$. Conversely, suppose that $M \subset X$ is a maximal $\mathcal{F}$-chain transitive set in $X$. In the same way, we show that $M$ is an $\mathcal{F}$-chain transitive set in $\beta X$. Hence, there is a maximal $\mathcal{F}$-chain transitive set $N \subset \beta X$ with $M \subset N$. Since $N \cap X$ is a maximal $\mathcal{F}$-chain transitive set in $X$ and $M \subset N \cap X$, it follows that $M=N \cap X$.

The main consequence of Theorem 1 is the required description for the chain recurrence set of $(S, X)$ in terms of attractors, as follows.

Theorem 2. The $\mathcal{F}$-chain recurrence set of $(S, X)$ is the set $\mathfrak{R}_{X}=\mathfrak{R} \cap X$, where $\mathfrak{R}$ is the $\mathcal{F}$-chain recurrence set of $(S, \beta X)$. In particular, one has

$$
\mathfrak{R}_{X}=\bigcap\left\{\mathcal{A} \cup \mathcal{A}^{*}: \mathcal{A} \text { is (an induced) } \mathcal{F} \text {-attractor in } X\right\} \text {. }
$$

Proof. Since the $\mathcal{F}$-chain recurrence set of $(S, X)$ is the union of all maximal $\mathcal{F}$ chain transitive sets in $X$, the theorem follows from Propositions 2, 3, and Theorem 1 .

Corollary 1. There is a finite number of maximal $\mathcal{F}$-chain transitive sets for $(S, X)$ if there is a finite one for $(S, \beta X)$. The semigroup action $(S, X)$ is $\mathcal{F}$ chain transitive if, and only if, the extended semigroup action $(S, \beta X)$ is $\mathcal{F}$-chain transitive.

An $\mathcal{F}$-chain control set for $(S, X)$ is a maximal $\mathcal{F}$-chain transitive set with nonempty interior. Chain control sets for semigroup actions have been introduced in [1. They considered the specific case where $S$ is a subsemigroup of a Lie group $G$ and $X$ is a homogeneous space of $G$. The notion of a chain control set for a control system has been extensively studied by Colonius and Kliemann (see [7] and the references therein).

Let $M \subset X$ be an $\mathcal{F}$-chain control set for $(S, X)$ and $U \subset \beta X$ an open set such that $\operatorname{int}_{X}(M)=U \cap X$. Let $N \subset \beta X$ be the maximal $\mathcal{F}$-chain transitive set such that $M=N \cap X$. Then, $U \cap X \subset N$. Since $N$ is closed and $X$ is dense in $\beta X$, it follows that $U \subset N$. Hence, $N$ is an $\mathcal{F}$-chain control set for $(S, \beta X)$. On the other hand, if $N \subset \beta X$ is an $\mathcal{F}$-chain control set, we have $\emptyset \neq \operatorname{int}(N) \cap X \subset \operatorname{int}_{X}(N \cap X)$. Hence, $N \cap X$ is an $\mathcal{F}$-chain control set in $X$. Thus, we have the following specific version of Theorem 1 .

Theorem 3. A nonempty subset $M \subset X$ is an $\mathcal{F}$-chain control set for $(S, X)$ if, and only if, there is an $\mathcal{F}$-chain control set $N$ for $(S, \beta X)$ such that $M=N \cap X$. In particular, there is a finite number of $\mathcal{F}$-chain control sets in $X$ if, and only if, there is a finite one in $\beta X$.

\section{Shadowing Semigroups}

Another way of describing maximal chain transitive sets of a semigroup action $(S, X)$ is provided in terms of the action of shadowing semigroups. The methodology considers control sets of shadowing semigroups, since their orbits reproduce the chains of $(S, X)$. We use this methodology to study chain recurrence on noncompact homogeneous spaces. 
Let $\mathcal{S}$ be a local semigroup on a topological space $X$, that is, a semigroup of maps defined on open sets of $X$. Assume that the orbits of $\mathcal{S}$ are open sets of $X$. A subset $D \subset X$ is a control set for $\mathcal{S}$ if

(1) $\operatorname{int}(D) \neq \emptyset$,

(2) $D \subset \operatorname{cl}(\mathcal{S} x)$ for all $x \in D$ and

(3) $D$ is maximal satisfying the last two properties.

The open set

$$
D_{0}=\{x \in D: x \in \mathcal{S} x\}
$$

is called the transitivity set of $D$. When $D_{0}$ is nonempty, $D$ is called an effective control set for $\mathcal{S}$. In this case, $D_{0}=\mathcal{S} x \cap \mathcal{S}^{*} x$, for all $x \in D_{0}$, and it is dense in $D$.

Given an open covering $\mathcal{U}$ of $X$, we define the $\mathcal{S}$-neighborhood of the identity map of $X$ relative to $\mathcal{U}$ as

$$
\mathcal{N}_{\mathcal{S}, \mathcal{U}}=\{\phi \in \mathcal{S}: \forall x \in \operatorname{dom} \phi, \exists U \in \mathcal{U} \text { such that } x, \phi(x) \in U\} .
$$

Let $(S, X, \mu)$ be an action of a semigroup $S$ on $X$. The shadowing semigroups are perturbations of $(S, X)$ by $\mathcal{S}$, as follows.

Definition 8. For all open coverings $\mathcal{U}$ of $X$ and $A \subset S$, we define the set

$$
\mathcal{N}_{\mathcal{S}, \mathcal{U}} A=\left\{\phi \mu_{s}: \phi \in \mathcal{N}_{\mathcal{S}, \mathcal{U}}, s \in A\right\}
$$

The $(\mathcal{U}, A)$-shadowing semigroup $\mathcal{S}_{\mathcal{U}, A}$ is the local semigroup generated by $\mathcal{N}_{\mathcal{S}, \mathcal{U}} A$.

The orbits of the shadowing semigroups describe trajectories with jumps in open sets of a covering, which are just chains of $(S, X)$.

Definition 9. Let $\mathcal{O}$ be an admissible family of open coverings of $M$. We say that $\mathcal{S}$ is $\mathcal{O}$-locally transitive if given a covering $\mathcal{U} \in \mathcal{O}$ and $U \in \mathcal{U}$, for every $x, y \in U$ there is $\phi \in \mathcal{N}_{\mathcal{S}, \mathcal{U}}$ such that $\phi(x)=y$.

For example, given $\mathcal{U} \in \mathcal{O}, U \in \mathcal{U}$ and $x \in U$, define $\phi_{U, x}: U \rightarrow M$ by $\phi_{U, x}(y)=x$. Then the local semigroup $\mathcal{S}=\left\{\phi_{U, x}: U \in \mathcal{U}, \mathcal{U} \in \mathcal{O}, x \in U\right\}$ is $\mathcal{O}$ locally transitive. In particular, the local semigroup $C_{l}(M)$ of all continuous maps defined on open subsets is $\mathcal{O}$-locally transitive.

The following result presents the link between the chains of $(S, M)$ and the action of the shadowing semigroups. It has been done in the setting of semiflows on topological spaces (see [13]). Here, we reproduce that result to semigroup actions.

Proposition 5. Given $x \in X$, an open covering $\mathcal{U}$ of $X$ and $A \subset S$, one has $\mathcal{S}_{\mathcal{U}, A} x \subset \Omega(x, \mathcal{U}, A)$ and $\left(\mathcal{S}_{\mathcal{U}, A}\right)^{*} x \subset \Omega^{*}(x, \mathcal{U}, A)$. Let $\mathcal{O}$ be an admissible family of open coverings of $X$. If $\mathcal{S}$ is $\mathcal{O}$-locally transitive, then $\mathcal{S}_{\mathcal{U}, A} x=\Omega(x, \mathcal{U}, A)$ and $\mathcal{S}_{\mathcal{U}, A}^{*} x=\Omega^{*}(x, \mathcal{U}, A)$, for all $\mathcal{U} \in \mathcal{O}$ and $A \subset S$.

Proof. Take $y \in \mathcal{S}_{\mathcal{U}, A} x$ and $\psi \in \mathcal{S}_{\mathcal{U}, A}$ such that $\psi(x)=y$. Write $\psi=$ $\phi_{n} \sigma_{a_{n}} \ldots \phi_{0} \sigma_{a_{0}}$, with $\phi_{i} \in \mathcal{N}_{\mathcal{S}, \mathcal{U}}$ and $a_{i} \in A, i=0, \ldots, n$. Denote $x_{0}=x, x_{1}=$ $\phi_{0}\left(a_{0} x_{0}\right), x_{2}=\phi_{1}\left(a_{1} x_{1}\right), \ldots, x_{n}=\phi_{n}\left(a_{n} x_{n}\right)=y$ in $X$. Then, for each $i$ there is $U_{i} \in \mathcal{U}$ such that $a_{i} x_{i}, \phi_{i}\left(a_{i} x_{i}\right) \in U_{i}$, that is, $a_{i} x_{i}, x_{i+1} \in U_{i}$. Hence, $y \in$ $\Omega(x, \mathcal{U}, A)$, whence $\mathcal{S}_{\mathcal{U}, A} x \subset \Omega(x, \mathcal{U}, A)$. Now, if $z \in\left(\mathcal{S}_{\mathcal{U}, A}\right)^{*} x$, then $x \in \mathcal{S}_{\mathcal{U}, A} z$. Hence, $x \in \Omega(z, \mathcal{U}, A)$ and $z \in \Omega^{*}(x, \mathcal{U}, A)$. Thus, $\left(\mathcal{S}_{\mathcal{U}, A}\right)^{*} x \subset \Omega^{*}(x, \mathcal{U}, A)$. For the second part of the proposition, assume that $\mathcal{S}$ is $\mathcal{O}$-locally transitive. Let 
$y \in \Omega(x, \mathcal{U}, A)$. Take $x_{0}=x, \ldots, x_{n}=y \in X, a_{0}, \ldots, a_{n-1} \in A$ and $U_{0}, \ldots, U_{n-1} \in \mathcal{U}$ such that $x_{i} a_{i}, x_{i+1} \in U_{i}, i=0, \ldots, n-1$. For each $i$ there is $\phi_{i} \in N_{\mathcal{S}, \mathcal{U}}$ such that $\phi_{i}\left(a_{i} x_{i}\right)=x_{i+1}$. Then, $y=\phi_{n-1} \sigma_{a_{n-1}} \ldots \phi_{0} \sigma_{a_{0}}(x) \in \mathcal{S}_{\mathcal{U}, A} x$. Hence, $\Omega(x, \mathcal{U}, A) \subset$ $\mathcal{S}_{\mathcal{U}, A} x$. Now, if $z \in \Omega^{*}(x, \mathcal{U}, A)$, then $x \in \Omega(z, \mathcal{U}, A) \subset \mathcal{S}_{\mathcal{U}, A} z$. Hence $z \in\left(\mathcal{S}_{\mathcal{U}, A}\right)^{*} x$, whence $\Omega^{*}(x, \mathcal{U}, A) \subset\left(\mathcal{S}_{\mathcal{U}, A}\right)^{*} x$. The proposition is proved.

From Proposition 5 we have the characterization of the maximal chain transitive sets as intersections of transitivity sets for the actions of shadowing semigroups.

Theorem 4. Let $\mathcal{O}$ be an admissible family of open coverings of $X$ and $\mathcal{F}$ a family of subsets of $S$. Assume that $\mathcal{S}$ is $\mathcal{O}$-locally transitive. Let $\mathcal{M} \subset X$ be a nonempty subset. Then the following condition is necessary and sufficient for $\mathcal{M}$ to be a maximal $\mathcal{F}$-chain transitive set:

- For all shadowing semigroups $\mathcal{S}_{\mathcal{U}, A}, \mathcal{U} \in \mathcal{O}$ and $A \in \mathcal{F}$, there is an effective control set $D_{\mathcal{U}, A}$ of $\mathcal{S}_{\mathcal{U}, A}$ such that

$$
\mathcal{M}=\bigcap_{\mathcal{U} \in \mathcal{O}, A \in \mathcal{F}}\left(D_{\mathcal{U}, A}\right)_{0}=\bigcap_{\mathcal{U} \in \mathcal{O}, A \in \mathcal{F}} D_{\mathcal{U}, A}=\bigcap_{\mathcal{U} \in \mathcal{O}, A \in \mathcal{F}} \operatorname{cls}\left(D_{\mathcal{U}, A}\right) .
$$

Proof. Take $x \in \mathcal{M}$. Then $\mathcal{S}_{\mathcal{U}, A} x=\Omega(x, \mathcal{U}, A)$ and $\mathcal{S}_{\mathcal{U}, A}^{*} x=\Omega^{*}(x, \mathcal{U}, A)$, for all $\mathcal{U} \in \mathcal{O}$ and $A \subset \mathcal{F}$. Since the sets $\Omega(x, \mathcal{U}, A)$ and $\Omega^{*}(x, \mathcal{U}, A)$ are open in $X$, the orbits $\mathcal{S}_{\mathcal{U}, A} x$ and $\mathcal{S}_{\mathcal{U}, A}^{*} x$ are open in $X$. Hence

$$
\begin{aligned}
\mathcal{M} & =\Omega_{\mathcal{O}, \mathcal{F}}(x) \cap \Omega_{\mathcal{O}, \mathcal{F}}^{*}(x) \\
& =\left\{\bigcap_{\mathcal{U} \in \mathcal{O}, A \in \mathcal{F}} \Omega(x, \mathcal{U}, A)\right\} \cap\left\{\bigcap_{\mathcal{U} \in \mathcal{O}, A \in \mathcal{F}} \Omega^{*}(x, \mathcal{U}, A)\right\} \\
& =\bigcap_{\mathcal{U} \in \mathcal{O}, A \in \mathcal{F}} \mathcal{S}_{\mathcal{U}, A} x \cap \mathcal{S}_{\mathcal{U}, A}^{*} x \\
& =\bigcap_{\mathcal{U} \in \mathcal{O}, A \in \mathcal{F}}\left(D_{\mathcal{U}, A}\right)_{0} .
\end{aligned}
$$

The other equalities follow since $\left(D_{\mathcal{U}, A}\right)_{0}$ is dense in $D_{\mathcal{U}, A}$.

4.1. Noncompact homogeneous spaces. Shadowing semigroups are very special tools for studying semigroup actions on homogeneous spaces. This is due to the fact that the topology of a homogeneous space is especially appropriate for constructing shadowing semigroups.

Let $G$ be a topological group, with $\mathcal{V}$ a basis of symmetric neighborhoods at the identity $e$ of $G$. For each $V \in \mathcal{V}$, define the open covering of $G$ :

$$
\mathcal{U}_{V}=\{V g: g \in G\} \text {. }
$$

Let $\mathcal{O}$ be the family of all open coverings $\mathcal{U}_{V}, V \in \mathcal{V}$. This family is admissible. For showing this fact, we need the following lemma.

Lemma 1. Let $K \subset N$ be subsets of $G$, with $K$ compact and $N$ open. There is $V \in \mathcal{V}$ such that $V K \subset N$.

Proof. For each $x \in K$, take $V_{x} \in \mathcal{V}$ such that $V_{x}^{2} \subset N$. The open covering $K \subset \bigcup_{x \in K} V_{x} x$ admits a finite subcovering $K \subset \bigcup_{i=1}^{n} V_{x_{i}} x_{i}$. Set $V=\bigcap_{i=1}^{n} V_{x_{i}} \in \mathcal{V}$. If $x \in K$, then $x \in V_{x_{i}} x_{i}$ for some $i$. It follows that $V x \subset V_{x_{i}}^{2} x_{i} \subset N$. Hence $V K \subset N$. 
Proposition 6. The family $\mathcal{O}$ of open coverings of $G$ is admissible.

Proof. For $\mathcal{U}_{V} \in \mathcal{O}$, take $U \in \mathcal{V}$ such that $U^{2} \subset V$. If $U g_{1}, U g_{2} \in \mathcal{U}_{U}$, with $U g_{1} \cap U g_{2} \neq \emptyset$, take $g \in U g_{1} \cap U g_{2}$. Then, there are $u_{1}, u_{2} \in U$ such that $g=u_{1} g_{1}=u_{2} g_{2}$. For $u \in U$, we have

$$
\begin{aligned}
& u g_{1}=u u_{1}^{-1} u_{1} g_{1}=u u_{1}^{-1} g \in U^{2} g \subset V g, \\
& u g_{2}=u u_{2}^{-1} u_{2} g_{2}=u u_{2}^{-1} g \in U^{2} g \subset V g .
\end{aligned}
$$

Hence, $U g_{1} \cup U g_{2} \subset V g$, where $V g \in \mathcal{U}_{V}$. Thus, $\mathcal{U}_{U} \leqslant \frac{1}{2} \mathcal{U}_{V}$. Now, let $Y \subset G$ be an open set and $K$ a compact subset of $G$ contained in $Y$. By Lemma 1, there is $V \in \mathcal{V}$ such that $V K \subset Y$. Take $U \in \mathcal{V}$ with $U^{2} \subset V$. If $U g \in\left[\mathcal{U}_{U}, K\right]$, there is $u \in U$ such that $u g \in K$. Then

$$
U g=U u^{-1} u g \subset U^{2} K \subset V K \subset Y .
$$

Hence, $\mathcal{U}_{U} \in \mathcal{O}$ is $K$-subordinated to $Y$. Finally, for any $\mathcal{U}_{V}, \mathcal{U}_{U} \in \mathcal{O}, \mathcal{U}_{V \cap U} \in \mathcal{O}$ is a refinement of both $\mathcal{U}_{V}$ and $\mathcal{U}_{U}$.

From now on, we assume that $G$ is a locally compact group and $H$ is a closed subgroup of $G$. Let $S \subset G$ be a semigroup and consider the semigroup action $(S, G / H)$. Let $\mathcal{F}$ be a family of subsets of $S$. The idea is to construct shadowing semigroups of $(S, G / H)$ by using the group $G$. Certainly, this is an advantage of studying the semigroup action on homogeneous spaces.

Proposition 7. Let $\pi: G \rightarrow G / H$ be the projection. For each $V \in \mathcal{V}$, define the open covering of $G / H$ by

$$
\pi \mathcal{U}_{V}=\{\pi(V g): g \in G\} .
$$

The family $\mathcal{O}_{\pi}=\left\{\pi \mathcal{U}_{V}: V \in \mathcal{V}\right\}$ of open coverings of $G / H$ is admissible. Furthermore, the group $G$ is $\mathcal{O}_{\pi}$-locally transitive.

Proof. For $\pi \mathcal{U}_{V} \in \mathcal{O}_{\pi}$, take $U \in \mathcal{V}$ such that $\mathcal{U}_{U} \leqslant \frac{1}{2} \mathcal{U}_{V}$. If $\pi\left(V g_{1}\right) \cap \pi\left(V g_{2}\right) \neq \emptyset$, there is $h \in H$ such that $V g_{1} \cap V g_{2} h \neq \emptyset$. Hence, there is $U g \in \mathcal{U}_{U}$ such that $V g_{1} \cup V g_{2} h \subset U g$. It follows that $\pi\left(V g_{1}\right) \cup \pi\left(V g_{2}\right) \subset \pi(U g)$. Thus, $\pi \mathcal{U}_{U} \leqslant \frac{1}{2} \pi \mathcal{U}_{V}$. Let $Y \subset G / H$ be an open set and $K$ a compact subset of $G / H$ contained in $Y$. Since $G$ is locally compact, there is a compact set $F \subset G$ with $\pi(F)=K$. There is $\mathcal{U}_{V} \in$ $\mathcal{O}$ that is $F$-subordinated to $\pi^{-1}(Y)$. If $\pi(V g) \in\left[\pi \mathcal{U}_{V}, K\right]$, then $V g h \in\left[\mathcal{U}_{V}, F\right]$ for some $h \in H$. It follows that $V g h \subset \pi^{-1}(Y)$, that is, $\pi(V g) \subset Y$. Hence, $\pi \mathcal{U}_{V}$ is $K$-subordinated to $Y$. Finally, for any $\pi \mathcal{U}_{V}, \pi \mathcal{U}_{U} \in \mathcal{O}_{\pi}$, it is immediate $\pi \mathcal{U}_{V \cap U} \in \mathcal{O}_{\pi}$ is a refinement of both $\pi \mathcal{U}_{V}$ and $\pi \mathcal{U}_{U}$. Now, we have $V \subset \mathcal{N}_{G, \pi \mathcal{U}_{V}}$ for all $V \in \mathcal{V}$. In fact, for $g H \in G / H$ and $v \in V$, we have $g H, v g H \in \pi(V g)$. Given a covering $\pi \mathcal{U}_{V} \in \mathcal{O}_{\pi}$ and $\pi(V g) \in \pi \mathcal{U}_{V}$, for $a H, b H \in \pi(V g)$, there are $v_{1}, v_{2} \in V$ such that $a H=v_{1} g H$ and $b H=v_{2} g H$. Hence, $v_{2} v_{1}^{-1} a H=b H$, with $v_{2} v_{1}^{-1} \in V$.

In particular, the shadowing semigroups $G_{\pi \mathcal{U}_{V}, A}$ have interior points in $G$.

Now, we are able to describe the chain transitivity of $(S, G / H)$ from Theorem 4 .

Theorem 5. A nonempty subset $\mathcal{M} \subset G / H$ is a maximal $\mathcal{F}$-chain transitive set if, and only if, for all shadowing semigroups $G_{\mathcal{U}, A}, \mathcal{U} \in \mathcal{O}_{\pi}$ and $A \in \mathcal{F}$, there is an effective control set $D_{\mathcal{U}, A}$ of $G_{\mathcal{U}, A}$ such that

$$
\mathcal{M}=\bigcap_{\mathcal{U} \in \mathcal{O}_{\pi}, A \in \mathcal{F}}\left(D_{\mathcal{U}, A}\right)_{0} .
$$


4.2. Adjoint orbits. Regular adjoint orbits of semisimple Lie groups are the most interesting noncompact spaces that we know of. Maximal chain transitive sets are parameterized by the Weyl group, which is finite.

Let $G$ be a connected noncompact semi-simple Lie group with finite center. Let $\mathfrak{g}$ be the Lie algebra of $G$ and $\mathfrak{g}=\mathfrak{k} \oplus \mathfrak{a} \oplus \mathfrak{n}$ an Iwasawa decomposition of $\mathfrak{g}$. Consider the noncompact homogeneous space $G / M A$, where $M A$ is the centralizer of $\mathfrak{a}$ in $G$. This homogeneous space identifies with any regular adjoint orbit in $\mathfrak{g}$ (see [15]). Moreover, the control sets for the action of a semigroup with nonempty interior $\mathcal{S} \subset G$ on $G / M A$ are parameterized by the Weyl group $W$ of $\mathfrak{g}$. Then, we can apply Theorem 5 to parameterize the maximal chain transitive sets of semigroup actions on $G / M A$.

In [15. Theorem 3.7] the effective control sets for the action of $\mathcal{S}$ on $G / M A$ were described by means of the Weyl group. In this description one has a mapping $w \rightarrow D(w)$ which associates to $w \in W$ a control set $D(w)$ in such a way that the set of transitivity $D(w)_{0}$ is the set of the fixed points of type $w$ for the split-regular elements in $\operatorname{int}(\mathcal{S})$.

Let $(S, G / M A)$ be a semigroup action, where $S \subset G$, and $\mathcal{F}$ a family of subsets of $S$. Let $\mathcal{O}_{\pi}$ be the admissible family of open coverings of $G / M A$ constructed as above.

Theorem 6. For each $w \in W$, set

$$
\mathcal{M}(w)=\bigcap_{\mathcal{U} \in \mathcal{O}_{\pi}, A \in \mathcal{F}}\left(D_{\mathcal{U}, A}(w)\right)_{0},
$$

where $D_{\mathcal{U}, A}(w)$ is the effective control set of $G_{\mathcal{U}, A}$ of type $w$. A nonempty subset $\mathcal{M} \subset G / M A$ is a maximal $\mathcal{F}$-chain transitive set if, and only if, $\mathcal{M}=\mathcal{M}(w)$ for some $w \in W$. In particular, the number of maximal chain transitive sets in $G / M A$ is finite.

Proof. Since the shadowing semigroups $G_{\mathcal{U}, A}$ have interior point in $G$, the theorem follows from Theorem 5 .

\section{REFERENCES}

[1] Braga Barros, C. J. and San Martin, L. A. B. (1996). Chain control sets for semigroup actions. Mat. Apl. Comp. 15, 257-276. MR1429552 (98b:93036)

[2] Braga Barros, C. J. and San Martin, L. A. B. (2007). Chain transitive sets for flows on flag bundles. Forum Math. 19, 19-60. MR2296065 (2008a:37019)

[3] Braga Barros, C. J. and Souza, J. A. (2010). Attractors and chain recurrence for semigroup actions. J. Dyn. Diff. Equations 22, 723-740. MR2734477 (2011k:37026)

[4] Braga Barros, C. J. and Souza, J. A. (2010). Finest Morse decompositions for semigroup actions on fiber bundles. J. Dyn. Diff. Equations 22, 741-760. MR2734478 (2011k:37027)

[5] Braga Barros, C. J., Souza, J. A. and Reis, R. A. (2012). Dynamic Morse decompositions for semigroups of homeomorphisms and control systems. J. Dyn. Control Syst. 18, 1-19.

[6] Choi, S. K., Chu, C.-K. and Park, J. S. (2002). Chain recurrent sets for flows on non-compact spaces. J. Dyn. Diff. Equations 14, 597-612. MR1917652 (2003d:37056)

[7] Colonius, F. and Kliemann, W. (2000). The Dynamics of Control. Birkhäuser, Boston. MR1752730 (2001e:93001)

[8] Hurley, M. (1991). Chain recurrence and attraction in non-compact spaces. Ergod. Th. Dynam. Syst. 11, 709-729. MR 1145617 (93b:58096)

[9] Hurley, M. (1992). Noncompact chain recurrence and attraction. Proc. Amer. Math. Soc. 115, 1139-1148. MR 1098401 (92j:58063)

[10] Hurley, M. (1995). Chain recurrence, semiflows, and gradients. J. Dyn. Diff. Equations 7, 437-456. MR1348735 (96k:58190) 
[11] Hurley, M. (1998). Lyapunov functions and attractors in arbitrary metric spaces. Proc. Amer. Math. Soc. 126, 245-256. MR.1458880 (98i:58147)

[12] Patrão, M. M. A. (2007). Morse decomposition of semiflows on topological spaces. J. Dyn. Diff. Equations 19, 181-198. MR.2279951 (2007m:37033)

[13] Patrão, M. M. A. and San Martin, L. A. B. (2007). Semiflows on topological spaces: chain transitivity and semigroups. J. Dyn. Diff. Equations 19, 155-180. MR2279950 (2007m:37032)

[14] Patrão, M. M. A. and San Martin, L. A. B. (2007). Morse decomposition of semiflows on fiber bundles. Discrete and Continuous Dynamical Systems (Series A) 17, 113-139. MR 2276428 (2008g:37010)

[15] Verdi, M. A., Rocio, O. G. and San Martin, L. A. B. (2009). Semigroup Actions on Adjoint Orbits. To appear.

[16] Willard, S. (2004). General topology. Dover Publications, New York. MR2048350

Departamento de Matemática, Universidade Estadual de Maringá, Maringá 87020900, BRASIL

E-mail address: jasouza3@uem.br 